\title{
ESTIMATES OF HARDY-RELLICH CONSTANTS FOR POLYHARMONIC OPERATORS AND THEIR GENERALIZATIONS
}

\author{
F.G. AVKHADIEV
}

\begin{abstract}
We prove the lower bounds for the functions introduced as the maximal constants in the Hardy and Rellich type inequalities for polyharmonic operator of order $m$ in domains in a Euclidean space. In the proofs we employ essentially the known integral inequality by O.A. Ladyzhenskaya and its generalizations. For the convex domains we establish two generalizations of the known results obtained in the paper M.P. Owen, Proc. Royal Soc. Edinburgh, 1999 and in the book A.A. Balinsky, W.D. Evans, R.T. Lewis, The analysis and geometry of hardy's inequality, Springer, 2015. In particular, we obtain a new proof of the theorem by M.P. Owen for polyharmonic operators in convex domains. For the case of arbitrary domains we prove universal lower bound for the constants in the inequalities for $m$ th order polyharmonic operators by using the products of $m$ different constants in Hardy type inequalities. This allows us to obtain explicit lower bounds for the constants in Rellich type inequalities for the dimension two and three. In the last section of the paper we discuss two open problems. One of them is similar to the problem by E.B. Davies on the upper bounds for the Hardy constants. The other problem concerns the comparison of the constants in Hardy and Rellich type inequalities for the operators defined in three-dimensional domains.
\end{abstract}

Keywords: polyharmonic operator, Hardy inequality, Rellich inequality, convex domain.

Mathematics Subject Classification: 26D15, 26D10

\section{INTRODUCTION}

Let $\Omega$ be a domain in the Euclidean space $\mathbb{R}^{d}(d \geqslant 2)$. We assume that $\Omega \neq \mathbb{R}^{d}$ and hence, the distance $\operatorname{dist}(x, \partial \Omega)$ from a point $x \in \Omega$ to the boundary of the domain is well-defined. We shall consider real-valued functions $f \in C_{0}^{\infty}(\Omega)$, that is, smooth functions $f: \Omega \rightarrow \mathbb{R}$ such that $\operatorname{supp}(f) \subset \Omega$. A functional $c_{2}(s, \Omega)$ introduced as the sharp constant in the following Hardy type inequality

$$
\int_{\Omega} \frac{|\nabla f(x)|^{2}}{(\operatorname{dist}(x, \partial \Omega))^{s-2}} d x \geqslant c_{2}(s, \Omega) \int_{\Omega} \frac{f^{2}(x)}{(\operatorname{dist}(x, \partial \Omega))^{s}} d x \quad \forall f \in C_{0}^{\infty}(\Omega)
$$

is well-studied, where $s \in(1, \infty)$ is a fixed parameter. In particular, a series of authors proved independently that $c_{2}(2, \Omega)=\frac{1}{4}$ for each convex domain $\Omega \neq \mathbb{R}^{d}$ (see [1]-[7]). If $s \in(1, d]$, there exist non-convex domains $\Omega^{\prime} \subset \mathbb{R}^{d}$ for which $c_{2}\left(s, \Omega^{\prime}\right)=0$, that is, the considered inequality is meaningless. There are various conditions ensuring the positivity of the constant $c_{2}(2, \Omega)$ (see, for instance, [3], [8]). In particular, it is well-known that $c_{2}(2, \Omega)>0$ for each bounded domain with a locally Lipschitz boundary.

F.G. Avkhadiev, Estimates of Hardy-Rellich Constants for polyharmonic operators and THEIR GENERALIZATIONS.

(C) Avkhadiev F.G. 2017.

The work is supported by RFBR (grant no. 17-01-00282-a).

Submitted June 14, $201 \%$. 
Let $m$ be a fixed natural number. Given a smooth real-valued function $f$, we consider the linear combinations of its partial derivatives of order $m$ introduced by the formulae:

$$
\Delta^{m / 2} f(x):= \begin{cases}\Delta^{j} f(x), & \text { if } m=2 j \text { is even; } \\ \nabla \Delta^{j} f(x), & \text { if } m=2 j+1 \text { is odd. }\end{cases}
$$

Here, as usually, $\Delta$ stands for the Laplace operator and $\nabla f$ is the gradient of the function $f$. It is obvious that $\Delta^{\frac{m}{2}}$ is a polyharmonic operator for even $m$.

We shall make use of the standard expression

$$
\left|D^{m} f(x)\right|^{2}:=\sum_{k_{1}=1}^{d} \sum_{k_{2}=1}^{d} \cdots \sum_{k_{m}=1}^{d}\left(\frac{\partial^{m} f(x)}{\partial x_{k_{1}} \partial x_{k_{2}} \cdots \partial x_{k_{m}}}\right)^{2}
$$

involving only the squares of partial derivatives of order $m$.

It should be noted that there are many works on Rellich inequalities for harmonic and polyharmonic operators as $m \geqslant 2, \Omega=\mathbb{R}^{d} \backslash\{0\}$ and weight functions are the powers of $|x|$ (see [7] [11] and the references therein). We shall consider the analogues of such inequalities as $\Omega \subset \mathbb{R}^{d}$ is a bounded or an unbounded domain and the weight functions are the powers of $\operatorname{dist}(x, \partial \Omega)$.

The aim of the present paper is the studying of the following inequality extending (1) for the case of the polyharmonic operators

$$
\int_{\Omega}\left|\Delta^{m / 2} f(x)\right|^{2} d x \geqslant A_{m}(\Omega) \int_{\Omega} \frac{f^{2}(x) d x}{(\operatorname{dist}(x, \partial \Omega))^{2 m}} \quad \forall f \in C_{0}^{\infty}(\Omega),
$$

where the function $\Delta^{m / 2} f$ is defined by formula (2), the constant $A_{m}(\Omega) \in[0, \infty)$ is the maximal possible, that is,

$$
A_{m}(\Omega)=\inf _{f \in C_{0}^{\infty}(\Omega), f \neq 0} \frac{\int_{\Omega}\left|\Delta^{m / 2} f(x)\right|^{2} d x}{\int_{\Omega} f^{2}(x)(\operatorname{dist}(x, \partial \Omega))^{-2 m} d x} .
$$

Moreover, we consider the following generalization of (1):

$$
\int_{\Omega} \frac{\left|D^{m} f(x)\right|^{2} d x}{(\operatorname{dist}(x, \partial \Omega))^{\sigma}} \geqslant C_{m}(\sigma, \Omega) \int_{\Omega} \frac{f^{2}(x) d x}{(\operatorname{dist}(x, \partial \Omega))^{2 m+\sigma}} \quad \forall f \in C_{0}^{\infty}(\Omega),
$$

where $\sigma \in(-1, \infty)$ is a fixed number, the function $\left|D^{m} f\right|^{2}$ is defined by formula $(3)$ and the constant $C_{m}(\sigma, \Omega) \in[0, \infty)$ is maximal, that is,

$$
C_{m}(\sigma, \Omega)=\inf _{f \in C_{0}^{\infty}(\Omega), f \neq 00} \frac{\int_{\Omega}\left|D^{m} f(x)\right|^{2}(\operatorname{dist}(x, \partial \Omega))^{-\sigma} d x}{\int_{\Omega} f^{2}(x)(\operatorname{dist}(x, \partial \Omega))^{-2 m-\sigma} d x} .
$$

We note that for the case $m \geqslant 2$, inequality (4) was studied just in several papers, while, to the best of the author's knowledge, inequality (5) was not studied before for the case $m \geqslant 2$ and $\sigma \neq 0$.

Inequality (4) was considered first by M.P. Owen [12]. He proved that for each $m \in \mathbb{N}$, $m \geqslant 2$, and each convex domain $\Omega \neq \mathbb{R}^{d}$ the estimate

$$
A_{m}(\Omega) \geqslant \frac{((2 m-1) ! !)^{2}}{4^{m}}
$$

holds true and this estimate is sharp since for the half-space $x_{1}>0$ it becomes the identity.

Some generalization and strengthening of the result by M.P. Owen were obtained in papers [13] [18]. In particular, we proved (see [17] and [18]) that $A_{2}(\Omega)=\frac{9}{16}$ for each convex domain $\Omega \neq \mathbb{R}^{d}$. 


\section{Simplest Properties of the CONStants AND SOME KNOWn FaCts}

It is obvious that as $m=1$, inequalities (4), (5) are reduced to inequality (1) since

$$
\left|\Delta^{1 / 2} f(x)\right|^{2} \equiv\left|D^{1} f(x)\right|^{2} \equiv|\nabla f(x)|^{2},
$$

and the identities $A_{1}(\Omega)=c_{2}(2, \Omega)$ and $C_{1}(\sigma, \Omega)=c_{2}(\sigma+2, \Omega)$ hold. This is why the natural condition $m \geqslant 2$ arises in statements on inequalities (4), (5) and the constants $A_{m}(\Omega), C_{m}(\sigma, \Omega)$.

It is easy to check that the constants $A_{m}(\Omega), C_{m}(\sigma, \Omega)$ and $c_{2}(s, \Omega)$ are invariant w.r.t. linear conformal mappings of the domain, that is, for each $a \in \mathbb{R} \backslash\{0\}, b \in \mathbb{R}^{d}$ the identities

$$
A_{m}(\Omega)=A_{m}(a \Omega+b), \quad C_{m}(\sigma, \Omega)=C_{m}(\sigma, a \Omega+b)
$$

hold true and $c_{2}(s, \Omega)=c_{2}(s, a \Omega+b)$, where $a \Omega+b=\{a x+b: x \in \Omega\}$.

Let us formulate three theorems, which will be used in what follows. We consider the functions $u: \Omega \rightarrow \mathbb{R} \in C_{0}^{\infty}(\Omega)$ and we concern Hardy type inequalities (1).

Theorem A. (see [1], 2] for $s=2$ and [19] for $s \neq 2$ ). Let $\Omega \subset \mathbb{R}^{d}$ be a convex domain, $\Omega \neq \mathbb{R}^{d}$. If $s \in(1, \infty)$, then

$$
\int_{\Omega} \frac{|\nabla u(x)|^{2} d x}{(\operatorname{dist}(x, \partial \Omega))^{s-2}} \geqslant \frac{(s-1)^{2}}{4} \int_{\Omega} \frac{u^{2}(x) d x}{(\operatorname{dist}(x, \partial \Omega))^{s}} \quad \forall u \in C_{0}^{\infty}(\Omega) .
$$

Theorem B. (see [19]). Let $\Omega \subset \mathbb{R}^{d}$ be an arbitrary domain, $\Omega \neq \mathbb{R}^{d}$. If $s \in(d, \infty)$, then

$$
\int_{\Omega} \frac{|\nabla u(x)|^{2} d x}{(\operatorname{dist}(x, \partial \Omega))^{s-2}} \geqslant \frac{(s-d)^{2}}{4} \int_{\Omega} \frac{u^{2}(x) d x}{(\operatorname{dist}(x, \partial \Omega))^{s}} \quad \forall u \in C_{0}^{\infty}(\Omega) .
$$

A series of Hardy and Rellich type inequalities with sharp constants are characterized by the absence of extremal functions in appropriate Sobolev spaces turning inequalities (1) and (4) into the identities. This is why there is a chance to strengthen these inequalities via increasing the right hand side by an additional positive term. For instance, as we have mentioned above, $c_{2}(2, \Omega)=\frac{1}{4}$ for each convex domain $\Omega \neq \mathbb{R}^{d}$. Nevertheless, the following statement holds true.

Theorem C. (see [4]). Let $\Omega \subset \mathbb{R}^{d}$ be a convex domain and let

$$
\delta_{0}(\Omega)=\sup _{x \in \Omega} \operatorname{dist}(x, \partial \Omega) .
$$

If $\delta_{0}(\Omega)<\infty$, then for each function $u \in C_{0}^{\infty}(\Omega)$

$$
\int_{\Omega}|\nabla u(x)|^{2} d x \geqslant \frac{1}{4} \int_{\Omega} \frac{u^{2}(x) d x}{(\operatorname{dist}(x, \partial \Omega))^{2}}+\frac{\lambda_{0}^{2}}{\delta_{0}^{2}(\Omega)} \int_{\Omega} u^{2}(x) d x,
$$

where $\lambda_{0} \approx 0.940$ is the first positive root of the Lamb equation $J_{0}(\lambda)+2 \lambda J_{0}^{\prime}(\lambda)=0$ for the Bessel function of zero order.

\section{RELlich TyPE INEQUALITIES IN CONVEX DOMAINS}

Our main aim is to generalize Theorem $\mathrm{C}$ for the polyharmonic operators. We first introduce some useful formulae for the integrals $\int_{\Omega}\left|\Delta^{m / 2} f(x)\right|^{2} d x$.

Let $f \in C_{0}^{\infty}(\Omega)$ be an arbitrary real function, then for the functions $\Delta^{\frac{1}{2}} f:=\nabla f$ and $\Delta^{1} f:=\Delta f$ the following integral identities hold:

$$
\begin{aligned}
& \int_{\Omega}|\nabla f(x)|^{2} d x=\int_{\Omega} \sum_{k=1}^{d}\left(\frac{\partial f(x)}{\partial x_{k}}\right)^{2} d x \\
& \int_{\Omega}(\Delta f(x))^{2} d x=\int_{\Omega} \sum_{k=1}^{d} \sum_{j=1}^{d}\left(\frac{\partial^{2} f(x)}{\partial x_{k} \partial x_{j}}\right)^{2} d x .
\end{aligned}
$$


Identity (9) is obviously implied simply by the definition of the gradient of the function. Identity (10) seems to be obtained first by O.A. Ladyzhenskaya (see, for instance, [21, Ch. 2, Form. (6.26)]) and this is a non-trivial identity since

$$
(\Delta f(x))^{2}=\sum_{k=1}^{d} \sum_{j=1}^{d} \frac{\partial^{2} f(x)}{\partial x_{k}^{2}} \frac{\partial^{2} f(x)}{\partial x_{j}^{2}} \not \equiv \sum_{k=1}^{d} \sum_{j=1}^{d}\left(\frac{\partial^{2} f(x)}{\partial x_{k} \partial x_{j}}\right)^{2} .
$$

We shall need an analogue of formulae (9) and (10) for polyharmonic operators. The following statement holds.

Assume that $m \in \mathbb{N}, \Omega \subset \mathbb{R}^{d}$ is a domain. Then for each real-valued function $f \in C_{0}^{\infty}(\Omega)$ the identity

$$
\int_{\Omega}\left|\Delta^{m / 2} f(x)\right|^{2} d x=\int_{\Omega} \sum_{k_{1}=1}^{d} \sum_{k_{2}=1}^{d} \cdots \sum_{k_{m}=1}^{d}\left(\frac{\partial^{m} f(x)}{\partial x_{k_{1}} \partial x_{k_{2}} \cdots \partial x_{k_{m}}}\right)^{2} d x
$$

holds true, or, the same, the identity

$$
\int_{\Omega}\left|\Delta^{m / 2} f(x)\right|^{2} d x=\int_{\Omega} \sum_{|\alpha|=m} \frac{m !}{\alpha !}\left(\frac{\partial^{m} f(x)}{\partial x^{\alpha}}\right)^{2} d x
$$

where $\partial x^{\alpha}=\partial x_{1}^{\alpha_{1}} \partial x_{2}^{\alpha_{2}} \cdots \partial x_{d}^{\alpha_{d}}, \partial x_{k}^{0}:=1 ; \alpha=\left(\alpha_{1}, \alpha_{2}, \cdots, \alpha_{d}\right)$ is a multi-index, $\alpha_{k}$ are nonnegative integer not exceeding $m ;|\alpha|=\alpha_{1}+\alpha_{2}+\cdots+\alpha_{d}, \alpha !=\alpha_{1} ! \alpha_{2} ! \cdots \alpha_{d} !$

It is obvious that identity $(12)$ is implied by (11) since by applying simple formulae of combinatorics we arrive at the following identity

$$
\sum_{k_{1}=1}^{d} \sum_{k_{2}=1}^{d} \cdots \sum_{k_{m}=1}^{d}\left(\frac{\partial^{m} f(x)}{\partial x_{k_{1}} \partial x_{k_{2}} \cdots \partial x_{k_{m}}}\right)^{2} \equiv \sum_{|\alpha|=m} \frac{m !}{\alpha !}\left(\frac{\partial^{m} f(x)}{\partial x^{\alpha}}\right)^{2} .
$$

We observe that formula (11) is known and it was provided, for instance, in monograph [22, Ch. 2, Form. (2.12)].

As we shall confirm in what follows, formula (11) turns out to be useful for studying inequalities (4) for the polyharmonic operators since it allows us to represent $\int_{\Omega}\left|\Delta^{m / 2} f(x)\right|^{2} d x$ as the sum of the integrals of squares of partial derivatives of order $m$.

Our main result is provided below in Theorem 2. First we obtain an analogue of Theorem A for inequalities (5) considered in convex domains. We note that Theorem 1 is used essentially in the proof of Theorem 2 .

Theorem 1. Let $\Omega \subset \mathbb{R}^{d}$ be a convex domain, $\Omega \neq \mathbb{R}^{d}$. Assume that $\sigma \in(-1, \infty)$ is a fixed number, $m$ is a natural number, $m \geqslant 2$. Then for each real-valued function $f \in C_{0}^{\infty}(\Omega)$ the inequality

$$
\int_{\Omega} \frac{\left|D^{m} f(x)\right|^{2} d x}{(\operatorname{dist}(x, \partial \Omega))^{\sigma}} \geqslant \frac{\Gamma^{2}\left(m+\frac{\sigma}{2}+\frac{1}{2}\right)}{\Gamma^{2}\left(\frac{\sigma}{2}+\frac{1}{2}\right)} \int_{\Omega} \frac{f^{2}(x) d x}{(\operatorname{dist}(x, \partial \Omega))^{2 m+\sigma}}
$$

holds true, where $\Gamma$ is the Euler Gamma-function. Therefore, for each convex domain $\Omega \neq \mathbb{R}^{d}$ the inequality

holds true.

$$
C_{m}(\sigma, \Omega) \geqslant \frac{\Gamma^{2}\left(m+\frac{\sigma}{2}+\frac{1}{2}\right)}{\Gamma^{2}\left(\frac{\sigma}{2}+\frac{1}{2}\right)}
$$

Proof. Let $f$ be a real-valued function belonging to $C_{0}^{\infty}(\Omega)$. By the definition of $\left|D^{m} f\right|^{2}$ and formula (13), the identity

$$
\left|D^{m} f(x)\right|^{2} \equiv \sum_{k_{1}=1}^{d} \sum_{k_{2}=1}^{d} \cdots \sum_{k_{m-1}=1}^{d}\left|\nabla u_{k_{1} k_{2} \cdots k_{m-1}}(x)\right|^{2}
$$


holds true, where

$$
u_{k_{1} k_{2} \cdots k_{m-1}}=\frac{\partial^{m-1} f}{\partial x_{k_{1}} \partial x_{k_{2}} \cdots \partial x_{k_{m-1}}} .
$$

Applying inequality (6) with $s=\sigma+2$ to the function $u=u_{k_{1} k_{2} \cdots k_{m-1}} \in C_{0}^{\infty}(\Omega)$ and summing up over indices $k_{1}, k_{2}, \cdots, k_{m-1}$ and taking into consideration formula (15), we have

$$
\int_{\Omega} \frac{\left|D^{m} f(x)\right|^{2} d x}{(\operatorname{dist}(x, \partial \Omega))^{\sigma}} \geqslant \frac{(1+\sigma)^{2}}{4} \int_{\Omega} \sum_{k_{1}=1}^{d} \sum_{k_{2}=1}^{d} \cdots \sum_{k_{m-1}=1}^{d} \frac{u_{k_{1} k_{2} \cdots k_{m-1}}^{2}(x) d x}{(\operatorname{dist}(x, \partial \Omega))^{2+\sigma}}
$$

or, the same,

$$
\int_{\Omega} \frac{\left|D^{m} f(x)\right|^{2} d x}{(\operatorname{dist}(x, \partial \Omega))^{\sigma}} \geqslant \frac{(1+\sigma)^{2}}{4} \int_{\Omega} \frac{\left|D^{m-1} f(x)\right|^{2} d x}{(\operatorname{dist}(x, \partial \Omega))^{2+\sigma}} .
$$

Since $\left|D^{1} f\right|^{2}=|\nabla f|^{2}$, as $m=2$, inequality 17 is equivalent to the following

$$
\int_{\Omega} \frac{\left|D^{2} f(x)\right|^{2} d x}{(\operatorname{dist}(x, \partial \Omega))^{\sigma}} \geqslant \frac{(1+\sigma)^{2}}{4} \int_{\Omega} \frac{|\nabla f(x)|^{2} d x}{(\operatorname{dist}(x, \partial \Omega))^{2+\sigma}} .
$$

Estimating from below the integral in the right hand side of this inequality by applying (6) with $s=\sigma+4$ to the function $u=f$, we obtain

$$
\int_{\Omega} \frac{\left|D^{2} f(x)\right|^{2} d x}{(\operatorname{dist}(x, \partial \Omega))^{\sigma}} \geqslant \frac{(1+\sigma)^{2}(3+\sigma)^{2}}{4^{2}} \int_{\Omega} \frac{f^{2}(x) d x}{(\operatorname{dist}(x, \partial \Omega))^{4+\sigma}},
$$

which is equivalent to inequality (14) as $m=2$ thanks to the identity

$$
\frac{(1+\sigma)(3+\sigma)}{4}=\frac{\Gamma\left(\frac{\sigma}{2}+\frac{5}{2}\right)}{\Gamma\left(\frac{\sigma}{2}+\frac{1}{2}\right)} .
$$

If $m \geqslant 3$, we arrive at inequality (14) by iterations and employing inequality (17) and the convention $\left(D^{0} f, D^{0} f\right)=f^{2}$. Namely, applying (17) with the numbers $m$ and $\sigma$ replaced by the numbers $m-j$ and $\sigma+2 j$ as $j=1, \cdots, m-1$, we get the inequalities

$$
\int_{\Omega} \frac{\left|D^{m-j} f(x)\right|^{2} d x}{(\operatorname{dist}(x, \partial \Omega))^{\sigma+2 j}} \geqslant \frac{(2 j+1+\sigma)^{2}}{4} \int_{\Omega} \frac{\left|D^{m-j-1} f(x)\right|^{2} d x}{(\operatorname{dist}(x, \partial \Omega))^{2 j+2+\sigma}},
$$

where $\left|D^{0} f\right|^{2}:=f^{2}$. Applying these inequalities with $j=1, \cdots, m-1$ for estimating from below the integral in the right hand side (17), we obtain

$$
\int_{\Omega} \frac{\left|D^{m} f(x)\right|^{2} d x}{(\operatorname{dist}(x, \partial \Omega))^{\sigma}} \geqslant\left(\prod_{j=0}^{m-1} \frac{2 j+1+\sigma}{2}\right)^{2} \int_{\Omega} \frac{f^{2}(x) d x}{(\operatorname{dist}(x, \partial \Omega))^{2 m+\sigma}},
$$

which is equivalent to inequality (14) due to the identity

$$
\prod_{j=0}^{m-1} \frac{2 j+1+\sigma}{2}=\frac{\Gamma\left(m+\frac{\sigma}{2}+\frac{1}{2}\right)}{\Gamma\left(\frac{\sigma}{2}+\frac{1}{2}\right)} .
$$

The proof is complete.

Identity (12) and the definition of the constants $A_{m}(\Omega)$ and $C_{m}(\sigma, \Omega)$ show that $A_{m}(\Omega)=$ $C_{m}(0, \Omega)$ for each domain $\Omega \subset \mathbb{R}^{d}, \Omega \neq \mathbb{R}^{d}$.

Applying Theorem 1 with $\sigma=0$, in view of this remark and the identity

$$
\frac{\Gamma\left(m+\frac{1}{2}\right)}{\Gamma\left(\frac{1}{2}\right)}=\frac{(2 m-1) ! !}{2^{m}},
$$

as a corollary, we obtain the result by M.P. Owen [12]. 
Corollary 1. (see [12]). For each natural $m \geqslant 2$ and each convex domain $\Omega \subset \mathbb{R}^{d}\left(\Omega \neq \mathbb{R}^{d}\right)$ the inequality

$$
\int_{\Omega}\left|\Delta^{m / 2} f(x)\right|^{2} d x \geqslant \frac{((2 m-1) ! !)^{2}}{4^{m}} \int_{\Omega} \frac{f^{2}(x) d x}{(\operatorname{dist}(x, \partial \Omega))^{2 m}}
$$

holds true for all $f \in C_{0}^{\infty}(\Omega)$.

We note that the original proof by M.P. Owen was based on using the distance function by E.B. Davies (see [3]) and it changes substantially from ours.

The next statement was proved in [7] as $m=2$. It strengthens the result by M.P. Owen in the case, when the domain $\Omega$ is convex and has a finite inner radius $\delta_{0}(\Omega)$. We note at the same time that there exist unbounded convex domains satisfying the condition $\delta_{0}(\Omega)<\infty$. For instance, $\delta_{0}\left(\Omega^{\prime}\right)=\frac{1}{2}$ for the domain

$$
\Omega^{\prime}=\left\{\left(x_{1}, x_{2}, \cdots, x_{d}\right) \in \mathbb{R}^{d}: 0<x_{1}<1\right\} .
$$

Theorem 2. Let $m \in \mathbb{N}, m \geqslant 2$, and let $\Omega \subset \mathbb{R}^{d}$ be a convex domain with a finite inner radius $\delta_{0}(\Omega)$. Then for each real-valued function $f \in C_{0}^{\infty}(\Omega)$ the inequalities

$$
\begin{aligned}
& \int_{\Omega}\left|\Delta^{m / 2} f(x)\right|^{2} d x \geqslant \Phi_{m}(f)+\frac{\lambda_{0}^{2}}{\delta_{0}^{2}(\Omega)} \int_{\Omega}\left|\Delta^{(m-1) / 2} f(x)\right|^{2} d x \\
& \int_{\Omega}\left|\Delta^{m / 2} f(x)\right|^{2} d x \geqslant \sum_{j=1}^{m} \frac{\lambda_{0}^{2(j-1)} \Phi_{m-j+1}(f)}{\delta_{0}^{2(j-1)}(\Omega)}+\frac{\lambda_{0}^{2 m}}{\delta_{0}^{2 m}(\Omega)} \int_{\Omega} f^{2}(x) d x,
\end{aligned}
$$

where $\lambda_{0} \approx 0.940$ is the first positive root of the Lamb equation $J_{0}(\lambda)+2 \lambda J_{0}^{\prime}(\lambda)=0$ for the Bessle function of zero order and

$$
\Phi_{k}(f)=\frac{((2 k-1) ! !)^{2}}{4^{k}} \int_{\Omega} \frac{f^{2}(x) d x}{(\operatorname{dist}(x, \partial \Omega))^{2 k}} \quad(k=1,2, \cdots, m) .
$$

Proof. Let $f \in C_{0}^{\infty}(\Omega)$ be an arbitrary real-valued function. This function satisfies formula (15). It is obvious that the function $u_{k_{1} k_{2} \cdots k_{m-1}}$ defined by identity (16) belongs also to the family $C_{0}^{\infty}(\Omega)$. Applying inequality (8) of Theorem $\mathrm{C}$ to the function $u=u_{k_{1} k_{2} \cdots k_{m-1}}$, we get

$$
\begin{aligned}
& \int_{\Omega}\left|\nabla u_{k_{1} k_{2} \cdots k_{m-1}}(x)\right|^{2} d x \geqslant \frac{1}{4} \int_{\Omega} \frac{u_{k_{1} k_{2} \cdots k_{m-1}}^{2}(x) d x}{(\operatorname{dist}(x, \partial \Omega))^{2}} \\
& +\frac{\lambda_{0}^{2}}{\delta_{0}^{2}(\Omega)} \int_{\Omega} u_{k_{1} k_{2} \cdots k_{m-1}}^{2}(x) d x .
\end{aligned}
$$

Summing up these inequalities over the indices $k_{1}, k_{2}, \cdots, k_{m-1}$ and taking into considerations identities (11) - 13), (15), we obtain

$$
\begin{aligned}
& \int_{\Omega}\left|\Delta^{m / 2} f(x)\right|^{2} d x=\int_{\Omega}\left|D^{m} f(x)\right|^{2} d x \geqslant \frac{1}{4} \int_{\Omega} \frac{\left|D^{m-1} f(x)\right|^{2} d x}{(\operatorname{dist}(x, \partial \Omega))^{2}} \\
& +\frac{\lambda_{0}^{2}}{\delta_{0}^{2}(\Omega)} \int_{\Omega}\left|D^{m-1} f(x)\right|^{2} d x .
\end{aligned}
$$

By Theorem 1 applied for $m-1$ and $\sigma=2$ we have

$$
\int_{\Omega} \frac{\left|D^{m-1} f(x)\right|^{2} d x}{(\operatorname{dist}(x, \partial \Omega))^{2}} \geqslant \frac{((2 m-1) ! !)^{2}}{4^{m-1}} \int_{\Omega} \frac{f^{2}(x) d x}{(\operatorname{dist}(x, \partial \Omega))^{2 m}}=\Phi_{m}(f) .
$$

Therefore, we obtain the inequality

$$
\int_{\Omega}\left|D^{m} f(x)\right|^{2} d x \geqslant \Phi_{m}(f)+\frac{\lambda_{0}^{2}}{\delta_{0}^{2}(\Omega)} \int_{\Omega}\left|D^{m-1} f(x)\right|^{2} d x
$$

equivalent to (18). 
Let us prove main inequality (19). It is obvious that as $m=1$, inequality (20) coincides with inequality (8) in Theorem $\mathrm{C}$. This, for each $k \in \mathbb{N}$ the inequality

$$
\int_{\Omega}\left|D^{k} f(x)\right|^{2} d x \geqslant \Phi_{k}(f)+\frac{\lambda_{0}^{2}}{\delta_{0}^{2}(\Omega)} \int_{\Omega}\left|D^{k-1} f(x)\right|^{2} d x
$$

holds true. It is clear that we can estimate from below the second term in the right hand side of inequality (20) by applying inequality (21) with $k=m-1$. As a result we have

$$
\int_{\Omega}\left|D^{m} f(x)\right|^{2} d x \geqslant \Phi_{m}(f)+\frac{\lambda_{0}^{2}}{\delta_{0}^{2}(\Omega)} \Phi_{m-1}(f)+\frac{\lambda_{0}^{4}}{\delta_{0}^{4}(\Omega)} \int_{\Omega}\left|D^{m-2} f(x)\right|^{2} d x .
$$

If $m=2$, the proof of inequality $(19)$ is complete in view of identity $\left|D^{0} \mathrm{f}\right|^{2}=f^{2}$.

If $m \geqslant 3$, we continue the process employing inequality (21) with $k=m-2$ for estimating the integral $\int_{\Omega}\left|D^{m-2} f(x)\right|^{2} d x$ from below. It is obvious that in $m$ steps we arrive at inequality (19). This completes the proof of the theorem.

\section{ESTIMATES OF CONSTANTS FOR ARBITRARY DOMAINS}

Let $c_{2}(s, \Omega)$ be the Hardy constant defined in Introduction. We recall that

$$
c_{2}(s, \Omega)=C_{1}(s-2, \Omega) .
$$

We are going to estimate from below the constant $C_{m}(\sigma, \Omega)$ for an arbitrary $d$-dimensional domain obeying the only condition $\Omega \neq \mathbb{R}^{d}$. This condition ensures the well-definiteness of the distance $\operatorname{dist}(x, \partial \Omega)$ and is hence natural for inequalities (5).

Theorem 3. Let $m \in \mathbb{N}, m \geqslant 2$, and let $\Omega \subset \mathbb{R}^{d}$ be an arbitrary domain $\Omega \neq \mathbb{R}^{d}$. If $\sigma \in(-1, \infty)$, then

$$
C_{m}(\sigma, \Omega) \geqslant \prod_{j=1}^{m} c_{2}(2 j+\sigma, \Omega)
$$

In particular,

$$
A_{m}(\Omega) \geqslant \prod_{j=1}^{m} c_{2}(2 j, \Omega)
$$

If $\sigma \in(d-2, \infty)$, then

$$
C_{m}(\sigma, \Omega) \geqslant \frac{\prod_{j=1}^{m}(2 j+\sigma-d)^{2}}{4^{m}}
$$

Proof. We follow the main lines of the proof of Theorem 1 with needed changes.

Let $f \in C_{0}^{\infty}(\Omega)$ be a fixed real-valued function. We write identity 15 for this function. At the first step we apply general Hardy inequality (1) to the function $u=u_{k_{1} k_{2} \cdots k_{m-1}} \in C_{0}(\Omega)$ in formula (15). Letting $s-2=\sigma$ in inequality (1), we obtain

$$
\int_{\Omega} \frac{\left|\nabla u_{k_{1} k_{2} \cdots k_{m-1}}(x)\right|^{2}}{(\operatorname{dist}(x, \partial \Omega))^{\sigma}} d x \geqslant c_{2}(2+\sigma, \Omega) \int_{\Omega} \frac{u_{k_{1} k_{2} \cdots k_{m-1}}^{2}(x)}{(\operatorname{dist}(x, \partial \Omega))^{2+\sigma}} d x .
$$

Summing up these inequalities over the indices $k_{1}, k_{2}, \cdots, k_{m-1}$, in view of formula (15) we get

$$
\int_{\Omega} \frac{\left|D^{m} f(x)\right|^{2} d x}{(\operatorname{dist}(x, \partial \Omega))^{\sigma}} \geqslant c_{2}(2+\sigma, \Omega) \int_{\Omega} \frac{\left|D^{m-1} f(x)\right|^{2} d x}{(\operatorname{dist}(x, \partial \Omega))^{2+\sigma}}
$$

Arguing in the same way to estimate from below the integral in the right hand side of this inequality for the indices $m-1, m-2, \cdots, 1$ and corresponding numbers $\sigma+2, \sigma+4, \cdots, \sigma+$ $2 m-2$. It is easy to see that in $m$ step we arrive at the inequality

$$
\int_{\Omega} \frac{\left|D^{m} f(x)\right|^{2} d x}{(\operatorname{dist}(x, \partial \Omega))^{\sigma}} \geqslant\left(\prod_{j=1}^{m} c_{2}(2 j+\sigma, \Omega)\right) \int_{\Omega} \frac{f^{2}(x) d x}{(\operatorname{dist}(x, \partial \Omega))^{2 m+\sigma}} .
$$


The latter inequality implies estimate $(22)$ since $C_{m}(\sigma, \Omega)$ is defined as the maximal constant in inequality (5).

If $\sigma \in(d-2, \infty)$ and $j=1,2, \cdots, m$, then $2 j+\sigma>d$. Therefore,

$$
c_{2}(2 j+\sigma, \Omega) \geqslant \frac{(2 j+\sigma-d)^{2}}{4}
$$

by Theorem B. Hence, inequality (24) follows Theorem B and inequality (22).

Thanks to the identity $A_{m}(\Omega)=C_{m}(0, \Omega)$, by inequality (24) we obtain (23). The proof is complete.

Corollary 2. Let $m \in \mathbb{N}, m \geqslant 2$, and $\Omega \subset \mathbb{R}^{d}$ be an arbitrary domain, $\Omega \neq \mathbb{R}^{d}$. Then the following statements holds:

1) if $d=2$, then

$$
A_{m}(\Omega) \geqslant((m-1) !)^{2} c_{2}(2, \Omega)
$$

2) if $d=3$, then

$$
A_{m}(\Omega) \geqslant \frac{((2 m-3) ! !)^{2} c_{2}(2, \Omega)}{4^{m-1}} .
$$

Proof. Estimate (23) obviously implies the inequality $A_{m}(\Omega) \geqslant X_{m} c_{2}(2, \Omega)$, where $X_{m}=$ $\prod_{j=2}^{m} c_{2}(2 j, \Omega)$.

Let $d=2$, then $c_{2}(2 j, \Omega) \geqslant(j-1)^{2}$ for $j=2, \cdots, m$ by Theorem B. Therefore, $X_{m} \geqslant$ $((m-1) !)^{2}$ for two-dimensional domains.

If $d=3$, we apply again Theorem B to estimate from below the constant $c_{2}(2 j, \Omega)$ as $j \geqslant 2$. We have

$$
c_{2}(2 j, \Omega) \geqslant \frac{(2 j-3)^{2}}{4}
$$

as $j=2, \cdots, m$. This yields the desired lower bound for $A_{m}(\Omega)$ for three-dimensional domains.

The constant $c_{2}(2, \Omega)$ is well-studied and there a series of known estimate for this constant in terms of geometric characteristics of the domain $\Omega$ (see, for instance, 3], 7], 8], [17 - 20], [25]). This is why Corollary 2 allows one to obtain a series of effective lower bounds for the constant $A_{m}(\Omega)$ for two- and three-dimensional domains.

\section{ON UNSOLVED PROBLEMS}

The theory of multi-dimensional Hardy and Rellich type inequalities is intensively developed. The interest to these inequalities is due to various applications in mathematical physics and harmonic analysis (see, for instance, [7]-[9], [23]). We provide just two unsolved problems related to the foundations of the theory and having simple formulations.

1. On upper bound for the constant $A_{m}(\Omega)$.

It follows from the definition $A_{m}(\Omega)$ that

$$
A_{m}(\Omega) \leqslant \frac{\int_{\Omega}\left|\Delta^{m / 2} f(x)\right|^{2} d x}{\int_{\Omega} f^{2}(x)(\operatorname{dist}(x, \partial \Omega))^{-2 m} d x}
$$

for each function $f \in C_{0}^{\infty}(\Omega), f \not \equiv 0$. This is why $A_{m}(\Omega)<\infty$ for each domain $\Omega \neq \mathbb{R}^{d}$ for each $m \in \mathbb{N}$.

Conjecture 1. Let $d=2$ and $d=3$. Then for each natural $m$ and each domain $\Omega \subset \mathbb{R}^{d}$ $\left(\Omega \neq \mathbb{R}^{d}\right)$ the sharp estimate

holds.

$$
A_{m}(\Omega) \leqslant \frac{((2 m-1) ! !)^{2}}{4^{m}}
$$


We note that as $m=1$, that is, for $A_{1}(\Omega)=c_{2}(2, \Omega)$, such conjecture was proposed by E.B. Davies in 1995 (see [24]) but it is not proved yet.

2. On positivity criterions for $c_{2}(2, \Omega)$.

We consider the domain $\Omega \subset \mathbb{R}^{2}\left(\Omega \neq \mathbb{R}^{2}\right)$. By $M_{0}(\Omega)$ we denote Euclidean maximal modulus defined by the identity

$$
M_{0}(\Omega)=\frac{1}{2 \pi} \sup _{K} \ln \frac{R(K)}{r(K)}
$$

where the supremum is taken over the concentric annuli

$$
K=\left\{x \in \mathbb{R}^{2}: r(K)<\left|x-x_{K}\right|<R(K)\right\}
$$

such that $K \subset \Omega, 0<r(K)<R(K)<\infty, x_{K} \in \partial \Omega$. If a domain $\Omega^{\prime} \subset \mathbb{R}^{2}$ contains no such annuli, by the definition we let $M_{0}\left(\Omega^{\prime}\right)=0$.

It is clear that the inequality $M_{0}(\Omega)<\infty$ is a geometric condition for the domain $\Omega \subset \mathbb{R}^{2}$.

It is known (see [17]-[20], [25]) that for a domain $\Omega \subset \mathbb{R}^{2}\left(\Omega \neq \mathbb{R}^{2}\right)$

$$
A_{1}(\Omega)=c_{2}(2, \Omega)>0 \Longleftrightarrow A_{2}(\Omega)>0 \Longleftrightarrow M_{0}(\Omega)<\infty .
$$

In particular, $c_{2}\left(2, \mathbb{R}^{2} \backslash\{0\}\right)=0$ and $M_{0}\left(\mathbb{R}^{2} \backslash\{0\}\right)=\infty$.

It is clear that one can define $M_{0}(\Omega)$ for a domain $\Omega \subset \mathbb{R}^{3}$ similar to the two-dimensional case replacing the annuli $K$ by spherical layers $\left\{x \in \mathbb{R}^{3}: r(K)<\left|x-x_{K}\right|<R(K)\right\}$.

Since $c_{2}\left(2, \mathbb{R}^{3} \backslash\{0\}\right)=\frac{1}{4}>0, A_{2}\left(\mathbb{R}^{3} \backslash\{0\}\right)=9 / 16>0$ and $M_{0}\left(\mathbb{R}^{3} \backslash\{0\}\right)=\infty$, statement 25 is not true for three-dimensional domains. Unfortunately, it is unclear by what one should replace the inequalty $M_{0}(\Omega)<\infty$ for multi-dimensional domains. This is why we can propose just a cut-down version of 25 for three-dimensional domains.

We have $c_{2}(4, \Omega)>0$ for three-dimensional domain by Theorems $\mathrm{C}$ and $A_{2}(\Omega) \geqslant$ $c_{2}(2, \Omega) c_{2}(4, \Omega)$ for arbitrary domains by estimate $(23)$. Therefore, $c_{2}(2, \Omega)>0 \Longrightarrow A_{2}(\Omega)>0$ for three-dimensional domains. These facts and the analogy with the two-dimensional case allow us to formulate the following conjecture.

Conjecture 2. For each domain $\Omega \subset \mathbb{R}^{3}\left(\Omega \neq \mathbb{R}^{3}\right)$ the relations

$$
A_{1}(\Omega)=c_{2}(2, \Omega)>0 \Longleftrightarrow A_{2}(\Omega)>0
$$

hold.

The difficulties arising while studying Conjecture 1, 2 are standard for the theory of Hardy and Rellich type inequalities. First, it is impossible to employ the methods of classical variational calculus because of the absence of extremal functions providing the identities and second, it is impossible to employ the symmetrization methods from the theory of isoperimetric inequalities because of the presence of the weight functions being the powers of the distance function $\operatorname{dist}(x, \partial \Omega)$.

The author thanks the referee for the useful remarks.

\section{BIBLIOGRAPHY}

1. T. Matskewich, P.E. Sobolevskii. The best possible constant in a generalized Hardy's inequality for convex domains in $\mathbb{R}^{n} / /$ Nonl. Anal. 28:9, 1601-1610 (1997).

2. M. Marcus, V.J. Mitzel, Y. Pinchover. On the best constant for Hardy's inequality in $\mathbb{R}^{n} / /$ Trans. Amer. Math. Soc. 350:8, 3237-3250 (1998).

3. E.B. Davies. A Review of Hardy inequalities // The Maz'ya anniversary Collection. 2. Oper. Theory Adv. Appl. 110, 55-67 (1999).

4. F.G. Avkhadiev, K.-J. Wirths Unified Poincaré and Hardy inequalities with sharp constants for convex domains // Z. Angew. Math. Mech. 87:8-9, 632-642 (2007).

5. F.G. Avkhadiev, A. Laptev. Hardy inequalities for nonconvex domains // in "Around Research of Vladimir Maz'ya, I". Inter. Mathem. Series. Springer, New York. 11, 1-12 (2010). 
6. F.G. Avkhadiev. A geometric description of domains whose Hardy constant is equal to 1/4 // Izv. RAN. Ser. Matem. 78:5, 3-26 (2014). [Izv. Math. 78:5, 855-876 (2014).]

7. A.A. Balinsky, W.D. Evans, R.T. Lewis. The analysis and geometry of Hardy's inequality. Springer, Heidelberg (2015).

8. V.G. Maz'ya. Sobolev spaces. Leningrad, Izd. Leningr. Univ. (1985). [Grundlehren der Mathematischen Wissenschaften. 342. Springer, Berlin (2011).]

9. F. Rellich. Perturbation theory of eigenvalue problems. Gordon and Breach, New York (1969).

10. P. Caldiroli, R. Musina. Rellich inequalities with weights // Calc. Var. Part. Diff. Equat. 45:1-2, 147-164 (2012).

11. F. Gesztesy, L. Littlejohn. Factorizations and Hardy-Rellich-type inequalities // Preprint: arXiv: $1701.08929 \mathrm{v} 1$

12. M.P. Owen. The Hardy-Rellich inequality for polyharmonic operators // Proc. Royal Soc. Edinburgh. Ser. A. 129:4, 825-839 (1999).

13. M.G. Barbatis. Improved Rellich inequalities for the polyharmonic operator // Indiana Univ. Math. J. 55:4, 1401-1422 (2006).

14. M.G. Barbatis and A. Tertikas. On a class of Rellich inequalities // J. Comp. Appl. Math. 194:1, 156-172 (2006).

15. W.D. Evans and R.T. Lewis. Hardy and Rellich inequalities with remainders // J. Math. Ineq. 1:4, 473-490 (2007).

16. E. Berchio, D. Cassani and F. Gazzola. Hardy-Rellich inequalities with boundary remainder terms and applications // Manuscript. Math. 131:3-4, 427-458 (2010).

17. F.G. Avkhadiev. Rellich type inequalities in domains of the Euclidean space // Izv. VUZov. Matem. 1, 69-73 (2016). [Russ. Math. (Izvestiya VUZ. Matem.), 60:1, 60-63 (2016).]

18. F.G. Avkhadiev. Hardy-Rellich inequalities in domains of the Euclidean space // J. Math. Anal. Appl. 442:2, 469-484 (2016).

19. F.G. Avkhadiev. Hardy type inequalities in higher dimensions with explicit estimate of constants // Lobachevskii J. Math. 21, 3-31 (2006).

20. F.G. Avkhadiev. Hardy-Type inequalities on planar and spatial open sets // Trudy Matem. Inst. Steklova. 255, 8-18 (2006). [Proc. Steklov Inst. Math. 255, 2-12 (2006).]

21. O.A. Ladyzhenskaya. The boundary value problems of mathematical physics. Nauka, Moscow (1973). [Springer, New York (1985).]

22. F. Gazzola, H-Ch. Grunau, G. Sweers Polyharmonic boundary value problems. Lect. Notes in Math. Springer, Berlin (1991).

23. M. Reed, B. Simon. Methods of mathematical physics. Scattering theory. Academic Press, San Diego (1979).

24. E.B. Davies The Hardy constant // Quart. J. Math.Oxford Ser.(2). 46:2, 417-431 (1995).

25. F.G. Avkhadiev. Integral inequalities in domains of hyperbolic type and their applications // Matem. Sborn. 206:12, 3-28 (2015). [Sborn. Math. 206:12, 1657-1681 (2015).]

Farit Gabidinovich Avkhadiev,

Kazan Federal University,

Kremlevskaya str., 18,

420008, Kazan, Russia

E-mail: avkhadiev47@mail.ru 